\title{
Modelación y simulación del rendimiento del pasto estrella (C.nlemfuensis) bajo diferentes condiciones de manejo y escenarios climáticos
}

DOI: https://doi.org/10.33262/ap.v2i3.33

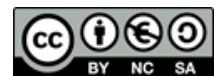

\section{Modeling and simulation of the performance of star grass (C. nlemfuensis) under different management conditions and climatic scenarios}

Jorge Lázaro López Rodríguez. ${ }^{1}$ \& Rubén Larduet Vicet. ${ }^{2}$

\begin{abstract}
.
The present work had as objectives to model and to simulate the production of accumulated dry matter (RMSA) of the grass it shatters (C.nlemfuensis) and to study the influence of two climatic scenarios on the acting of this variable and this way to contribute to the taking of decisions under unfavorable environmental conditions for the development of this cultivation. The considered scenarios were: half temperature of the planet rises $2^{\circ} \mathrm{C}$ (A2) and that this variable rises $4^{\circ} \mathrm{C}$ (B2). For it, information of experiments was used generated in areas of the Institute of Animal Science and of works published about the influence of the climate RMSA it has more than enough, it was found that the pattern Gompertz was that of better adjustment of the RMSA vs the sprout age, with high coefficients of determination, biases and small estimate errors in all the analyzed mensurations. They were also obtained a group of remarkable indicators for the taking of decisions of producers and investigating, such as inflection point (age of the cultivation in which their curve of growth passes of a type of concavity to other), good value of crop that corresponds to the value of the age in that you to intersect the curves of increments current means (RMSA divided among the age) and newspaper (derived of the function of Gompertz evaluated in the
\end{abstract}

\footnotetext{
${ }^{1}$ Aeropuerto Internacional José Martí, jorge.lopez@takeoff.avianet.cu

${ }^{2}$ Universidad Agraria de la Habana. La Habana, Cuba. jorge.lopez@takeoff.avianet.cu
} 
age), among others. The pattern exponential multiplicative was selected to study the effect of the climate RMSA it has more than enough, where it was appreciated that the studied scenarios A2 and B2, had negative effects on the yield of accumulated dry matter, because they caused increase of half temperature and decrease of the precipitations and global radiation. A computing program was obtained (SERPE) that estimates the accumulated yields of dry matter under different environmental conditions.

Keywords: modulation, simulation, accumulated dry matter, function of Gompertz, SERPE.

\section{Resumen.}

El presente trabajo tuvo como objetivos modelar y simular la producción de materia seca acumulada (RMSA) del pasto estrella (C. nlemfuensis) y estudiar la influencia de dos escenarios climáticos sobre el desempeño de esta variable y de esta forma contribuir a la toma de decisiones en condiciones ambientales desfavorables para el desarrollo de este cultivo. Los escenarios considerados fueron: temperatura media del planeta se eleve $2{ }^{\circ} \mathrm{C}$ (A2) y que esta variable se eleve $4{ }^{\circ} \mathrm{C}$ (B2). Para ello, se utilizó información de experimentos generados en áreas del Instituto de Ciencia Animal y de trabajos publicados acerca de la influencia del clima sobre RMSA, se encontró que el modelo Gompertz fue el de mejor ajuste del RMSA vs la edad de rebrote, con elevados coeficientes de determinación, sesgos y errores de estimación pequeños en todas las mediciones analizadas. Se obtuvieron además un grupo de indicadores notables para la toma de decisiones de productores e investigadores, tales como punto de inflexión (edad del cultivo en la cual su curva de crecimiento pasa de un tipo de concavidad a otra), valor óptimo de cosecha, que corresponde al valor de la edad en que se intersectan las curvas de incrementos corriente medio (RMSA dividida entre la edad) y diario (derivada de la función de Gompertz evaluada en la edad), entre otros. Fue seleccionado el modelo multiplicativo exponencial para estudiar el efecto del clima sobre RMSA, donde se apreció que los escenarios estudiados A2 y B2, tuvieron efectos negativos sobre el rendimiento de materia seca acumulada, debido a que ocasionaron aumento de temperatura media y disminución de las precipitaciones y radiación global. Se obtuvo un programa computacional (SERPE) que estima los rendimientos acumulados de materia seca bajo diferentes condiciones ambientales.

Palabras claves: modelación, simulación, materia seca acumulada, función de Gompertz, SERPE.

\section{Introducción.}

La ganadería se considera una fuente de progreso importante para el desarrollo económico por ser este un sector dinámico y en fuerte crecimiento (Friedrich, 2014). En Cuba, esta actividad cobra gran importancia con la actualización del modelo económico y social y se fundamenta en la implementación del lineamiento 136 del VI Congreso del Partido 
Comunista de Cuba (PCC) con mayor énfasis en la utilización de los pastos y forrajes en la alimentación del ganado vacuno (PCC, 2011).

Según Herrera (2009), un gran número de investigaciones estudian el comportamiento de los sistemas de producción de esos alimentos y su uso en la alimentación animal. Estos estudios son de especial interés para encontrar métodos cuantitativos que permitan la interpretación de los principales factores que afectan su producción y de esta forma desarrollar acciones que con lleven a maximizar las producciones sin alterar la sostenibilidad de los sistemas en el tiempo (Rodríguez et al., 2010).

Por otra parte, el cambio climático influye directamente sobre el crecimiento, desarrollo de plantas y cultivos, los balances hidrológicos, la frecuencia, tipo e intensidad de las siembras, así como en la severidad de la erosión de la tierra.

También afecta, entre otras variables, la disponibilidad y temporalidad de los sistemas de irrigación. Diversos estudios para distintas regiones y países han confirmado que el calentamiento global es ya un problema al que se debe prestar gran atención por sus repercusiones sobre el bienestar de los seres humanos. Los años de la última década han sido los más cálidos en los registros históricos de la temperatura mundial (PCCC, 2015) y hacia el futuro se espera que la Tierra siga presentando cambios climatológicos al modificarse los patrones de temperatura y precipitación. Algunos estudios (Adams et al., 1998), han argumentado que como resultado del cambio climático se tendrían efectos adversos sobre la seguridad alimentaria ya que los rendimientos de algunos cultivos importantes disminuirían al igual que la productividad pecuaria. De igual forma se reduciría la disponibilidad de agua tanto para la agricultura como para el consumo humano.

Hasta ahora se han realizado ciertos esfuerzos para tratar de enfrentar el problema del cambio climático. En mayo de 2015, los Estados miembros de la ONU, adoptaron la Convención Marco de las Naciones Unidas sobre Cambio Climático, con el objetivo último de lograr la estabilización de las concentraciones de los gases de efecto invernadero (GEI) en la atmósfera, a un nivel que impida interferencias antropógenas peligrosas en el sistema climático y en un plazo suficiente para permitir que los ecosistemas se adapten naturalmente al cambio climático, asegurando que la producción de alimentos no se vea amenazada y permita que el desarrollo económico prosiga de manera sostenible.

No obstante, aún con las políticas actuales de mitigación de los efectos del cambio climático y con las prácticas de desarrollo sostenible que aquellas conllevan, existe evidencia abundante respecto a que las emisiones mundiales de GEI seguirán aumentando en los próximos decenios En el caso que lo hicieran a una tasa igual o superior a la actual, el calentamiento global aumentaría y el sistema climático mundial experimentaría durante el siglo XXI cambios probablemente mayores que los observados durante el siglo XX (IPCC, 2015). 
Diferentes investigaciones han aportado evidencia de que los más afectados serán los países en vías de desarrollo, que soportarán aproximadamente entre el $75 \%$ y el $80 \%$ del costo de los daños provocados por la variación del clima Para América Latina y el Caribe, se ha encontrado que, si no se toman en cuenta los efectos de $\mathrm{CO} 2$, la reducción en la producción de granos podría ser hasta de $30 \%$ bajo el escenario de mayor temperatura. Sin embargo, si dichos efectos son tomados en consideración, la producción en algunos países podría incluso incrementarse. Centroamérica y el Caribe es una región altamente vulnerable a los fenómenos climáticos y meteorológicos dada su extensión y ubicación geográfica (Banco Mundial, 2015).

Periódicamente, esta región se ve afectada por huracanes e inundaciones que impactan negativamente el desarrollo y el bienestar de sus habitantes y el desempeño de las actividades económicas. Como se documenta en Leary et al. (2007), es muy probable que el cambio climático intensifique la gravedad de los fenómenos extremos, como los anteriores.

En este sentido, Cuba se debe preparar para enfrentar el desafío del cambio climático, de ahí que disponer de instrumentos que permitan simular situaciones caóticas para los cultivos, permitirá tomar medidas alternativas para mitigar los mismos.

Los efectos del cambio climático, han sido estudiados en las viandas, caña de azúcar, tabaco, entre otros cultivos (PCCC, 2015), pero no existen evidencias de su estudio en los pastos tropicales, específicamente en el pasto estrella (C.nlemfuensis).

La obtención de nuevos datos mediante la experimentación agronómica, además de generar resultados específicos para cada lugar en concreto en el espacio y el tiempo es un proceso costoso, lo que sugiere la búsqueda de otras alternativas para dar respuesta a tal situación, entre los que se enmarcan los modelos matemáticos y de simulación (Rodríguez, 2015).

Estos resultan herramientas de amplia utilización en los últimos años para lograr los objetivos propuestos. Los modelos matemáticos se utilizan en diferentes ramas del conocimiento por la posibilidad de expresar matemáticamente las relaciones entre los fenómenos (Jay, 2012). Mientras que los modelos de simulación parten de la necesidad de integrar los conocimientos en una herramienta computacional que permita entender el funcionamiento del sistema y sus componentes y faciliten la toma de decisiones acertadas (Hernández et al., 2009).

La modelación y simulación del pasto estrella (C. nlemfuensis), constituye vía efectiva para sistematizar la información, realizar proyecciones, programaciones en los sistemas de producción ganaderos y estudiar la influencia de las variables climáticas en el rendimiento de este cultivo (Agudelo et al., 2008).

Con todo lo anteriormente expuesto, el objetivo de esta investigación es modelar y simular el comportamiento productivo de la especie pasto estrella (C. nlemfuensis) bajo diferentes frecuencias de corte, niveles de fertilización y condiciones climatológicas en la producción 
de biomasa, que contribuya a la toma de decisiones, en condiciones climáticas adversas para el desarrollo de este cultivo.

\section{Materiales y métodos.}

Se utilizó la información proveniente de investigaciones desarrolladas durante un año en el centro experimental "Miguel Sistach Naya" perteneciente al Departamento de Pastos y Forrajes del Instituto de Ciencia Animal, situado en San José de las Lajas, provincia Mayabeque, Cuba, entre los $22^{\circ} 53^{\prime} \mathrm{LN}$ y los $82^{\circ} 02^{\prime} \mathrm{LW}$, a 92 msnm.

El experimento se desarrolló en un suelo Ferralítico Rojo compactado, bastante uniforme en todo su perfil y de rápida desecación (Anon, 1994). Se utilizó un área de pasto estrella previamente establecida, con más del $95 \%$ de pureza dividida en parcelas experimentales, con un área cosechable de $10 \mathrm{~m}^{2}$.

Durante el período experimental (mayo 1992-abril 1993) se aplicó una fertilización basal de 100 y 200 kgha-1 año-1 de P2O5 (superfosfato triple) y K2O (óxido de potasio), respectivamente y se distribuyó al 50\% al inicio de cada estación del año. El nitrógeno (nitrato de amonio) se fracciono a razón de 50 kgha-1 por cada ciclo de crecimiento (equivalente a $200 \mathrm{~kg} \mathrm{~N}$ ha-1 año-1), dosis en la cual se obtienen las mayores respuestas en términos de kg MS kg-1 N aplicado en los suelos rojos (Ramos et al., 1979; Ramos et al., 1995). En el período seco se aplicó riego por aspección a razón de 500 m3 ha-1 cada $21 \pm 3$ días.

Previo al inicio de cada ciclo experimental las parcelas se cortaron con una segadora para uniformar el rebrote y entre estos se ofreció un período mínimo de tiempo que garantizara, de conjunto con la distribución aleatoria de las edades dentro de cada réplica, que el corte de las parcelas se efectuara nunca antes de seis semanas de reposo en cada tratamiento entre ciclos de crecimiento.

Antes del corte en cada semana se cosecharon 10 plantas previamente marcadas al azar por tratamiento en cada réplica. En sus hojas se midió el largo desde la lígula hasta el ápice por el haz y el ancho en el punto medio longitudinal y en sus tallos, el largo del diámetro en los entrenudos definidos.

En el experimento original, se utilizó un diseño en parcelas divididas con tres replicas donde el nivel de $\mathrm{N}$ constituyó la parcela principal (0, 200 y $400 \mathrm{~kg}$ de $\mathrm{N}$ ha-1 año-1) y las subparcelas las edades desde 1 hasta 12 semanas.

\section{Análisis matemático.}

Se emplearon dos modelos de regresión no lineal cuyas expresiones son las siguientes:

Gompertz

$$
W_{(t)}=A e^{-b e^{-c t}}+\varepsilon
$$


Modelo multiplicativo con efectos

$$
W_{(t)}=A e^{\left(d t+f t^{2}+g t^{3}\right)} Y Z+\varepsilon
$$

ambientales (Del Pozo y Herrera 1995)

Dónde:

$W_{(t)}$ V Variable dependiente que representa la acumulación de biomasa en el tiempo.

A, b, c, d, f, g: son parámetros de los modelos.

t: es la variable independiente y representa el tiempo medido en días o semanas.

Y: efecto de la radiación.

Z: efecto de la precipitación. $\varepsilon$ : es error aleatorio, normalmente distribuido con media cero y varianza constante.

Para el modelo multiplicativo con efectos ambientales, se impusieron las siguientes restricciones adicionales (Del Pozo y Herrera 1995):

$$
\sum_{\mathrm{k}}^{n} \mathrm{Z}_{\mathrm{k}}=\sum_{\mathrm{j}}^{n} \mathrm{Y}_{\mathrm{j}}=1
$$

Se comprobó el cumplimiento de los supuestos teóricos del modelo de regresión según Montgomery et al. (2005).

El comportamiento de las tasas de acumulación de biomasa de la variedad se describió, mediante las curvas de evolución en el tiempo del incremento corriente diario (ICD) y del incremento medio diario (IMD) según Kiviste et al. (2002), las cuales se definen como:

$$
\begin{aligned}
& I C D=\frac{d W}{d t} \\
& I M D=\frac{W}{t}
\end{aligned}
$$

Se determinó el punto de inflexión y el momento adecuado para la utilización de la planta por medio de las ecuaciones siguientes:

$\frac{d^{2} W}{d t^{2}}=\left[\frac{\ln (b)}{c} ; \frac{a}{e}\right]$

Punto de inflexión

Momento de utilización de la planta

$$
t e^{-c t}=\frac{1}{b c}
$$


El porcentaje del valor asintótico del rendimiento (\% VA) para decidir hasta que edad sería razonable evaluar la curva de acumulación de la planta, se calculó como:

$$
\% V A=\frac{R M S A_{(\text {edad de rebrote })}}{A} * 100
$$

El procesamiento de la información se realizó con apoyo de los paquetes estadísticos InfoStat (Di Rienzo et al., 2012) y Statgraphics Plus (Anon, 1995).

Se utilizaron las ecuaciones de RMSA sin efectos ambientales obtenidas en el Capítulo 2 (Gompertz), el modelo multiplicativo obtenido por Del Pozo y Herrera (1995) dosis 200 y $400 \mathrm{~kg}$ de N.

Para los análisis de los contextos climáticos futuros, se usaron salidas diarias de los Modelos de Circulación General (MCG) Echan4 y se utilizó el forzamiento de los escenarios de emisiones (SRES, según sus siglas en inglés) A2 y B2 propuestos por el IPCC (2001) con una resolución espacial de $25 \mathrm{~km}$. Esta selección de los escenarios, la ratifica Bárcena (2010) y Bárcena et al (2014), ya que consideran que las condiciones de América Latina y el Caribe seguirán determinadas por el desarrollo económico, con nuevas tecnologías "limpias", principalmente a nivel de regiones o localidades. Además, este modelo que se utiliza, se encuentra entre los disponibles y representa, en forma más adecuada, la circulación general de la atmósfera en el Caribe, según criterios de Jones et al. (2004) y Campbell et al. (2011).

Echan4 herramienta principal para hacer proyecciones sobre el cambio climático en el futuro, emplea modelos físicos complejos. Analiza la gama completa de escenarios, se complementa con modelos físicos simples, calibrados para ofrecer una respuesta equivalente a los complejos, en cuanto a la temperatura y al nivel del mar. Estas proyecciones se obtienen a partir de un modelo simple del clima cuya sensibilidad climática y consumo calorífico de los océanos estén calibrados con cada uno de los siete modelos climáticos complejos. La sensibilidad climática que presenta el modelo simple varía entre 1,7 y $4,2^{\circ} \mathrm{C}$, similar a la variación comúnmente aceptada, que va de 1,5 a $4,5^{\circ} \mathrm{C}$.

Para esta investigación, se tomó como referencia, los valores medios de precipitación y temperatura del período 1961 - 1990, y se consideraron los valores medios proyectados de dichas variables para los horizontes temporales 2011 - 2040, 2041 - 2070 y 2071 - 2099 para los escenarios seleccionados.

Según el Panel Intergubernamental de Cambio Climático (IPCC son las siglas en inglés), los "escenarios" son descripciones coherentes y consistentes de cómo el sistema climático de la Tierra puede cambiar en el futuro. Estos son derivados de los escenarios de posibles emisiones futuras de GEI, los cuales se utilizan en modelos climáticos como elemento introducido para el cálculo de proyecciones climáticas. Cualquier descripción posible del 
clima futuro dependerá de cómo se asuman las emisiones futuras de los GEI y otros agentes contaminantes; es decir, dependerán de la opción del panorama de las emisiones.

Los escenarios del Reporte Especial de Escenarios de Emisiones (REEE) (IPCC, 2001) se utilizan como base de las proyecciones climáticas, de Modelo de Circulación General (MCG) y Modelos de Circulación General Acoplados de la Atmósfera y el Océano (MCGAO). Comprenden una línea evolutiva similar, en lo que respecta a los supuestos demográficos, sociales, económicos y de cambios tecnológicos y se constituyen por cuatro familias de escenarios: A2 y B2 (IPCC 2000; IPCC, 2001) las cuales se explican en la tabla 1.

Tabla 1. Características de los escenarios de emisiones propuestos por el IPCC.

\begin{tabular}{|c|c|}
\hline A2 & B2 \\
\hline \multicolumn{2}{|c|}{ Propone una situación donde el mundo no sigue un patrón globalizante } \\
\hline $\begin{array}{l}\text { Se conservan las identidades locales y la } \\
\text { población crece a un ritmo medio. El } \\
\text { desarrollo económico y tecnológico es } \\
\text { fragmentado y más lento que en otros } \\
\text { escenarios. }\end{array}$ & $\begin{array}{l}\text { Priman las soluciones locales para } \\
\text { problemáticas sociales, económicas y } \\
\text { ambientales. Está orientado a la protección } \\
\text { del medio ambiente y a la igualdad social, } \\
\text { pero siempre a nivel local o regional, }\end{array}$ \\
\hline $\begin{array}{l}\text { Se produce un aumento de la } \\
\text { temperatura global del planeta en } 2{ }^{\circ} \mathrm{C}\end{array}$ & $\begin{array}{l}\mathrm{Se} \quad \text { produce } \quad \text { un aumento de } \\
\text { temperatura global del planeta en } 4{ }^{\circ} \mathrm{C}\end{array}$ \\
\hline
\end{tabular}

Fuente: Elaboración propia.

Con esta información, se creó una base de datos. Como no existían datos sobre la radiación global, esta se estimó a partir de las fórmulas reportadas por la FAO (1997). Se seleccionó la estación meteorológica de Güines situada en los $22^{\circ} 53^{\prime \prime}$ latitud norte y $82^{\circ}$ longitud oeste provincia de Mayabeque por su cercanía a las áreas del Instituto de Ciencia Animal.

\section{Herramientas y tecnologías de desarrollo utilizadas.}

La selección de los entornos de trabajos que se utiliza en el desarrollo de sistemas, constituye un verdadero reto y un gran desafío para los analistas. Existen diferencias y por supuesto ventajas y desventajas entre las plataformas de desarrollo, motores de bases de datos y los lenguajes de programación existentes que los desarrolladores deben dominar para tomar una adecuada decisión en cada caso. Este proceso se debe realizar de forma tal que satisfagan los requerimientos al menor costo posible, sin que signifique una pérdida de funcionalidad y eficiencia en el producto a lograr.

\section{Lenguaje de Programación: Java.}


Según Paz (2011) "Java es una tecnología orientada al desarrollo de software con el cual se puede realizar cualquier tipo de programa”. Es estable, portable, sencillo y menos complejos que otros lenguajes de alto nivel.

Dentro de las principales características se establece que constituye un lenguaje de programación orientado a objetos y de gran flexibilidad a la hora de integrarse con disimiles tecnologías. Es independiente de la plataforma, lo que garantiza ejecución de las aplicaciones en múltiples plataformas de hardware y sistemas operativos (Unix, Linux, OS/390, Windows entre otros) (Puerto; 2012).

Para llevar a cabo el desarrollo de una aplicación guiada por el lenguaje mencionado, es necesario contar con alguna herramienta que lo soporte y permita desarrollar, compilar, ejecutar y probarla. Se puede constatar en las investigaciones llevadas a cabo por (Paz,2011; Puerto, 2012 y Páez,2012) que el uso del lenguaje de programación Java lleva asociado la aplicación de un Entorno de Desarrollo Integrado (IDE).

\section{Entorno de Desarrollo Integrado (IDE): Netbeans.}

Un Entorno de Desarrollo Integrado o IDE (acrónimo en inglés de integrated development environment) constituye un ambiente de programación que consta de en un editor de código, un compilador, un depurador y un constructor de interfaz gráfica (GUI). En la actualidad existen múltiples IDE para el desarrollo de software entre los que se encuentran MyEclipse, Eclipse y NetBeans los cuales son utilizados para el desarrollo de aplicaciones java (Travieso, 2015).

El Netbeans es un IDE de código abierto escrito completamente en Java. Soporta el desarrollo de todos los tipos de aplicación Java (J2SE, web y aplicaciones móviles). También tiene soporte para varios lenguajes como PHP, Java script, HTML y CSS. Es un producto sin restricciones de uso (Rodríguez, 2015).

El autor de esta investigación, determinó utilizar el Netbeans en su versión 8.0, ya que simplifica tareas de desarrollo, además del sencillo manejo de los componentes visuales de los formularios de la aplicación, así como diseños de reportes.

\section{Gestor de reportes: IReport.}

La gestión de reporte es el proceso que se utiliza para la toma de decisiones o bien para confeccionar informes detallados a los clientes, proveedores o bien sus propios empleados. Brindan la posibilidad de crear reportes según los parámetros que se especifican (Rodríguez, 2015a).

La herramienta iReport es un constructor y a su vez diseñador de informes visuales, poderoso, intuitivo y fácil de usar. Está escrito en Java y permite que los usuarios corrijan visualmente informes complejos con cartas, imágenes, sub-informes, entre otros. Brinda integración con 
JFreeChart, una de las bibliotecas gráficas Open Source más difundida para Java. Los datos para mostrar pueden ser recuperados de disímiles fuentes de datos (Travieso, 2015).

\section{Sistema Gestor de Bases de Datos: SQLite.}

García (2011) define los SGBD como "un conjunto de información almacenada en memoria auxiliar que permite acceso directo y un conjunto de programas que manipulan esos datos". Un SGBD es una aplicación que permite a los usuarios definir, crear y mantener dicha base, proporcionando un acceso controlado a la misma.

\section{Resultados y discusión}

En las tablas 2 y 3 se presentan los criterios estadísticos del modelo (Gompertz) en ambos períodos estacionales, para edades expresadas en días y semanas respectivamente.

Los modelos explicaron alta variabilidad entre el RMSA y las edades de rebrote, con valores superiores al $99 \%$ en el período lluvioso y poco lluvioso, en cada dosis de N (0 y 200). El CME, EE y Sesgo fueron pequeños lo cual repercutió en los elevados valores de significación de los modelos y sus parámetros.

Tabla 2. Estadísticos de ajuste y estimaciones de los parámetros de los modelos del rendimiento de materia seca acumulada (RMSA) con la edad de rebrote de la planta (en días)

\begin{tabular}{|c|c|c|c|c|c|c|c|c|c|}
\hline Período & $\begin{array}{c}\text { Dosis } \\
\mathbf{N}\end{array}$ & $\begin{array}{l}\text { R2 } \\
(\%)\end{array}$ & CME & $\mathbf{E E}$ & Sesgo & $\begin{array}{l}\text { Sign. } \\
\text { modelo }\end{array}$ & $\mathbf{A}$ & b & c \\
\hline \multirow[t]{6}{*}{ Lluvioso } & & & & & 0,007 & & 2,62 & 6,52 & 0,05 \\
\hline & 0 & 99,39 & 0,004 & 0,07 & & $\mathrm{P}<0,001$ & $\pm 0,13$ & $\pm 0,91$ & $\pm 0,0046$ \\
\hline & & & & & & & $\mathrm{P}<0,001$ & $\mathrm{P}<0,001$ & $\mathrm{P}<0,001$ \\
\hline & & & & & & & 6,06 & 4,55 & 0,05 \\
\hline & 200 & 99,90 & 0,005 & 0,06 & 0,004 & $\mathrm{P}<0,001$ & $\pm 0,1$ & $\pm 0,2$ & $\pm 0,0017$ \\
\hline & & & & & & & $\mathrm{P}<0,001$ & $\mathrm{P}<0,05$ & $\mathrm{P}<0,001$ \\
\hline Poco & & & & & & & 1,37 & 3,64 & 0,04 \\
\hline \multirow[t]{5}{*}{ lluvioso } & 0 & 99,57 & 0,001 & 0,03 & 0,001 & $\mathrm{P}<0,001$ & $\pm 0,03$ & $\pm 0,72$ & $\pm 0,0005$ \\
\hline & & & & & & & $\mathrm{P}<0,001$ & $\mathrm{P}<0,001$ & $\mathrm{P}<0,001$ \\
\hline & & & & & & & 3,92 & 3,81 & 0,03 \\
\hline & 200 & 99,34 & 0,007 & 0,08 & 0,004 & $\mathrm{P}<0,001$ & $\pm 0,33$ & $\pm 0,31$ & $\pm 0,004$ \\
\hline & & & & & & & $\mathrm{P}<0,001$ & $\mathrm{P}<0,001$ & $\mathrm{P}<0,001$ \\
\hline
\end{tabular}

Fuente: Elaboración propia.

Tabla 3. Estadísticos de ajuste y estimaciones de los parámetros de los modelos del rendimiento de materia seca acumulada (RMSA) con la edad de rebrote de la planta (en semanas) 
ISSN: 2773-7330

Vol. 2, $\mathrm{N}^{\circ} 3$, p. 21-42

julio-septiembre, 2020

\begin{tabular}{|c|c|c|c|c|c|c|c|c|c|}
\hline Período & Dosis N & $\begin{array}{l}\text { R2 } \\
(\%)\end{array}$ & CME & $\mathbf{E E}$ & Sesgo & $\begin{array}{c}\text { Sign. } \\
\text { modelo }\end{array}$ & A & b & c \\
\hline \multirow{6}{*}{ Lluvioso } & & & & & & & 2,62 & 6,52 & 0,33 \\
\hline & 0 & 99,42 & 0,005 & 0,07 & 0,007 & $\mathbf{P}<0,001$ & $\pm 0,13$ & $\pm 0,91$ & $\pm 0,03$ \\
\hline & & & & & & & $\mathrm{P}<0,001$ & $\mathbf{P}<0,001$ & $\mathbf{P}<0,001$ \\
\hline & & & & & & & 6,06 & 4,55 & 0,32 \\
\hline & 200 & 99,34 & 0,007 & 0,08 & 0,004 & $\mathrm{P}<0,001$ & $\pm 0,1$ & $\pm 0,2$ & $\pm 0,01$ \\
\hline & & & & & & & $\mathrm{P}<0,001$ & $\mathrm{P}<0,05$ & $\mathrm{P}<0,001$ \\
\hline \multirow{4}{*}{$\begin{array}{l}\text { Poco } \\
\text { 1luvioso }\end{array}$} & & & & & & & 1,37 & 3,64 & 0,3 \\
\hline & 0 & 99,32 & 0,04 & 0,20 & 0,001 & $\mathrm{P}<0,001$ & $\pm 0,05$ & $\pm 0,27$ & $\pm 0,02$ \\
\hline & & & & & & & $\mathbf{P}<0,001$ & $\mathrm{P}<0,001$ & $\mathrm{P}<0,001$ \\
\hline & 200 & 99,34 & 0,007 & 0,08 & 0,004 & $\mathrm{P}<0,001$ & $\begin{array}{c}3,92 \\
\pm 0,33 \\
\mathrm{P}<0,001\end{array}$ & $\begin{array}{c}3,81 \\
\pm 0,31 \\
\mathrm{P}<0,001\end{array}$ & $\begin{array}{c}0,22 \\
\pm 0,03 \\
\mathrm{P}<0,001\end{array}$ \\
\hline
\end{tabular}

Fuente: Elaboración propia.

En las figuras desde la 1 hasta 4, se muestran los gráficos de los residuos para las dosis $0 \mathrm{y}$ 200 de $\mathrm{N}$ y edades expresadas en días y semanas. Se aprecian que, en todos los casos, existen pocas desviaciones de los puntos respecto de la diagonal principal lo que confirma la hipótesis de normalidad de los residuos.

Figura 1: Gráficos para dosis 0 de $\mathrm{N}$ en seca y edades en días y semanas respectivamente.
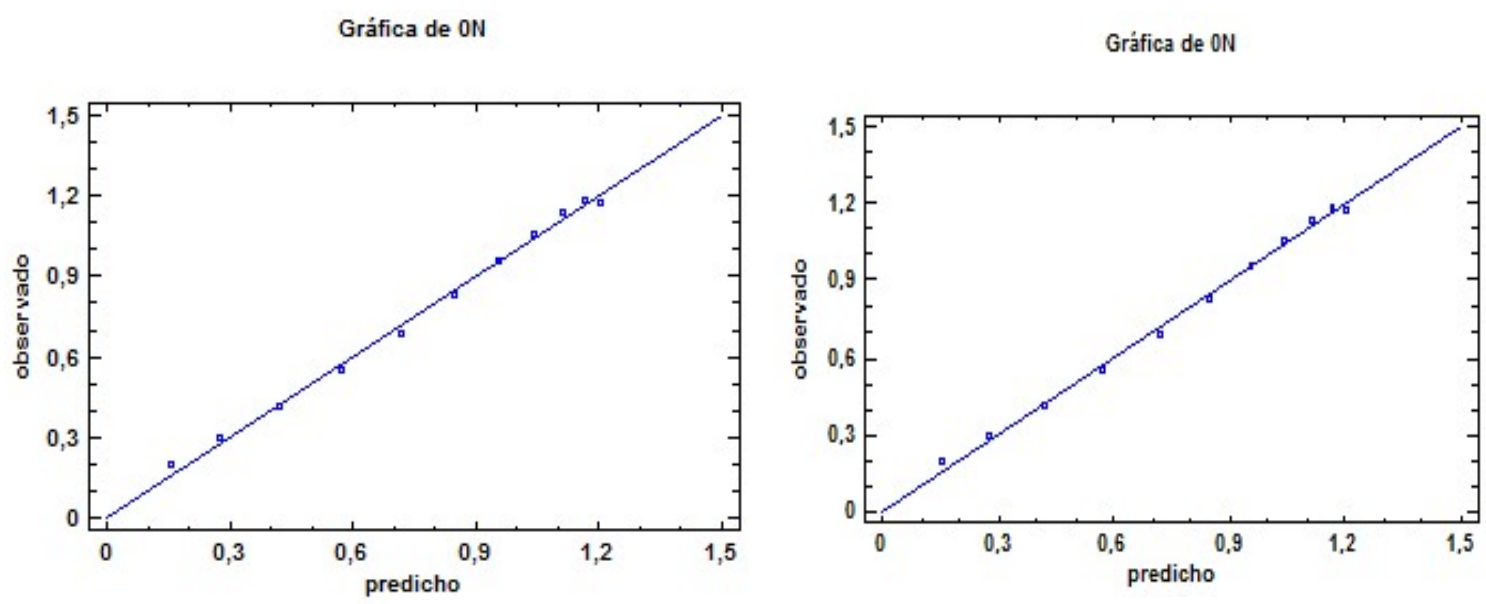

Fuente: Elaboración propia.

Figura 2: Gráficos para dosis 0 de $\mathrm{N}$ en lluvia y edades en días y semanas respectivamente. 
Grafica de ONLLuvia

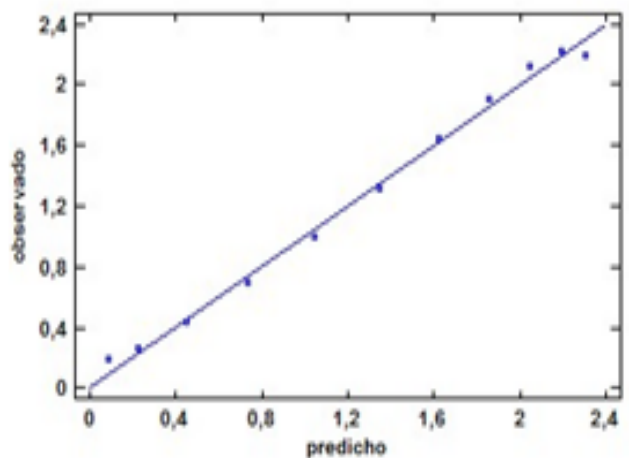

Gráfica de OHLLluvia

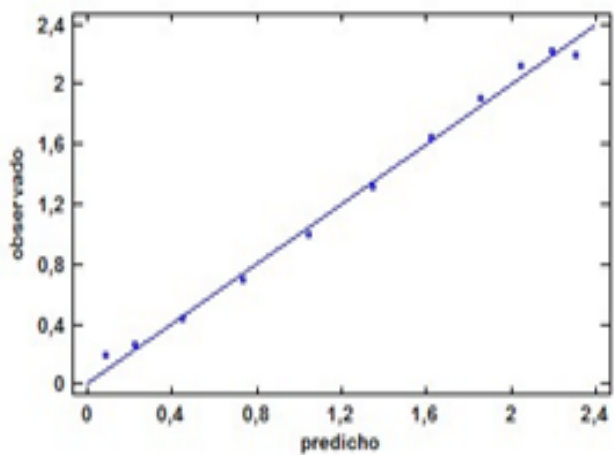

Fuente: Elaboración propia.

Figura 3. Gráficos para dosis 200 de $\mathrm{N}$ en lluvia y edades en días y semanas respectivamente.
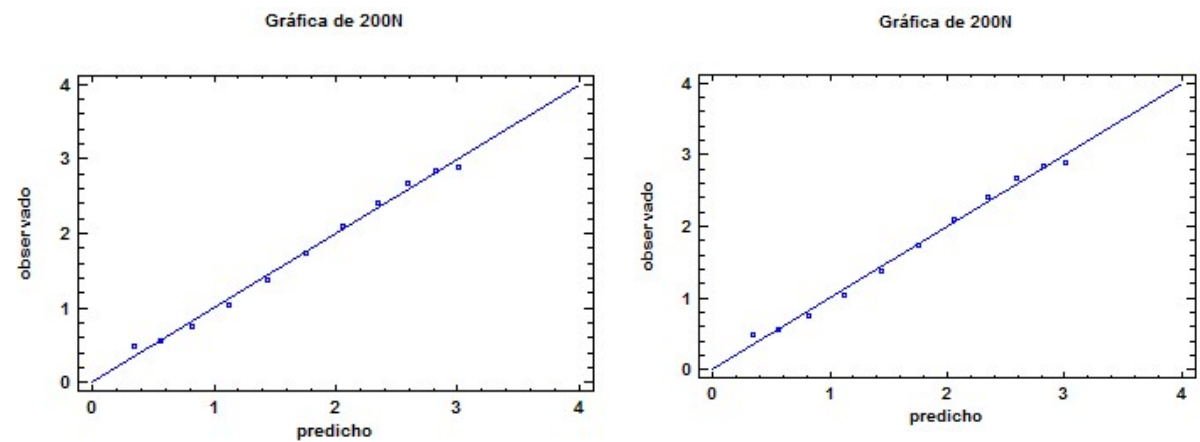

Fuente: Elaboración propia.

Figura 4. Gráficos para dosis 200 de $\mathrm{N}$ en seca y edades en días y semanas respectivamente.
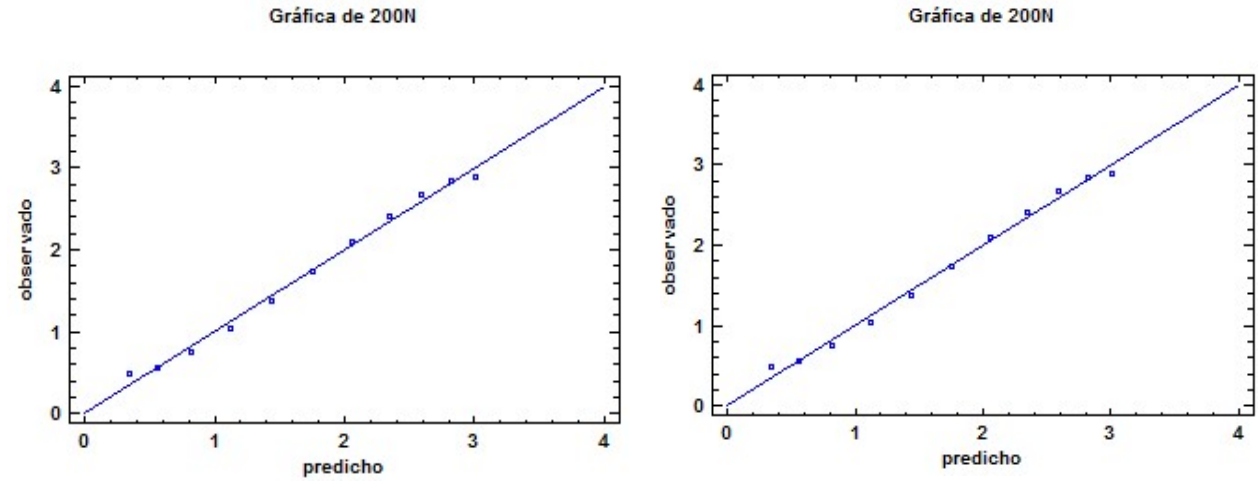

Fuente: Elaboración propia. 
Estas características confieren alta precisión a los modelos para representar el proceso de acumulación de biomasa del pasto estrella.

En cuanto a los parámetros en los dos períodos estacionales se observó que los valores de A y c fueron cercanos y sus errores estándares pequeño. Esto indicó menor amplitud de los intervalos de confianza de estos parámetros lo cual confiere mayor concentración de sus valores alrededor del verdadero valor del parámetro (Montgomery et al., 2005).

Como se apreció, en los resultados encontrados, el modelo de Gompertz representó de forma adecuada el crecimiento del pasto estrella, resultado que coincide con los encontrados por Díaz (2007) y Rodríguez (2015) en variedades de la especie P. purpureum y Barker et al. (2010) en otras gramíneas de sendero fotosintético C4.

Según Black (1973) Las plantas tipo C4 presentan las siguientes características:

- Además del ciclo fotosintético con intermediarios de 3 carbonos disponen de otro alternativo con intermediarios de 4 carbonos.

- Viven en climas tropicales (maíz, sorgo, caña de azúcar, entre otras).

- No se saturan y aprovechan las altas intensidades luminosas.

- Son todas plantas vasculares.

- Transpiran poco (Soportan bien la escasez de agua).

- Sus células tienen dos tipos de cloroplasto.

- Son especialistas de zonas cálidas y húmedas.

- Gastan 5 ATP para fijar un O2.

Estos últimos resaltaron la asimetría del modelo para representar el crecimiento de gramíneas tropicales perennes en un ciclo de 112 días o 12 semanas. Según estos autores las plantas C4 presentan tasas de incremento antes (aceleración) y después del punto de inflexión (desaceleración) diferentes, lo cual guarda relación lógica con el proceso de acumulación de biomasa de gramíneas tropicales.

En la tabla 4 se muestra la edad a la que se alcanzó el punto de inflexión, el punto óptimo para cortar el forraje y la fase L.V.C. o período de lenta velocidad de crecimiento, los cuales constituyen parámetros de interés para el desarrollo de estrategias en la investigación y la producción. 
Tabla 4. Relación entre la edad y el rendimiento en el punto de inflexión, punto óptimo y fase de lenta velocidad de crecimiento para las diferentes dosis de $\mathrm{N}$ en el período poco lluvioso y lluvioso.

\begin{tabular}{|c|c|c|c|c|c|}
\hline \multicolumn{6}{|c|}{ Poco lluvioso } \\
\hline \multirow{2}{*}{$\begin{array}{l}\text { Dosis N } \\
\left(\mathrm{kg} \mathrm{ha}^{-}\right. \\
\left.{ }^{1} \mathrm{anno}^{-1}\right)\end{array}$} & \multicolumn{2}{|c|}{$\begin{array}{l}\text { Punto de } \\
\text { Inflexión }\end{array}$} & \multicolumn{2}{|c|}{$\begin{array}{l}\text { Punto } \\
\text { Optimo }\end{array}$} & \multirow{2}{*}{$\begin{array}{l}\text { L.V.C } \\
\text { (días) }\end{array}$} \\
\hline & $\begin{array}{l}\text { Edad } \\
\text { (días) }\end{array}$ & $\begin{array}{l}\text { RMSA } \\
\left(\text { tha }^{-1}\right)\end{array}$ & $\begin{array}{l}\text { Edad } \\
\text { (días) }\end{array}$ & $\begin{array}{l}\text { RMSA } \\
\left(\text { tha }^{-1}\right)\end{array}$ & \\
\hline 0 & 84 & 1,21 & 32,3 & 0,50 & 7,30 \\
\hline 200 & 84 & 2,89 & 44,6 & 1,4 & 11,3 \\
\hline \multicolumn{6}{|c|}{ Lluvioso } \\
\hline Dosis N & \multicolumn{2}{|c|}{$\begin{array}{l}\text { Punto de } \\
\text { Inflexión }\end{array}$} & \multicolumn{2}{|c|}{$\begin{array}{l}\text { Punto } \\
\text { Optimo }\end{array}$} & L.V.C \\
\hline $\begin{array}{l}(\mathrm{kg} \mathrm{ha}- \\
\left.{ }^{1} \text { año }^{-1}\right)\end{array}$ & $\begin{array}{l}\text { Edad } \\
\text { (días) }\end{array}$ & $\begin{array}{l}\text { RMSA } \\
\left(\text { tha }^{-1}\right)\end{array}$ & $\begin{array}{l}\text { Edad } \\
\text { (días) }\end{array}$ & $\begin{array}{l}\text { RMSA } \\
\left(\text { tha }^{-1}\right)\end{array}$ & (días) \\
\hline 0 & 84 & 2,38 & 37,5 & 0,96 & 17,5 \\
\hline 200 & 84 & 5,66 & 30,3 & 2,2 & 10,3 \\
\hline
\end{tabular}

Fuente: Elaboración propia.

La figura 5 muestra la ventana principal de la aplicación desarrollada con cada uno de los principales elementos del menú.

Figura 5. Pantalla principal de la aplicación. SERPE

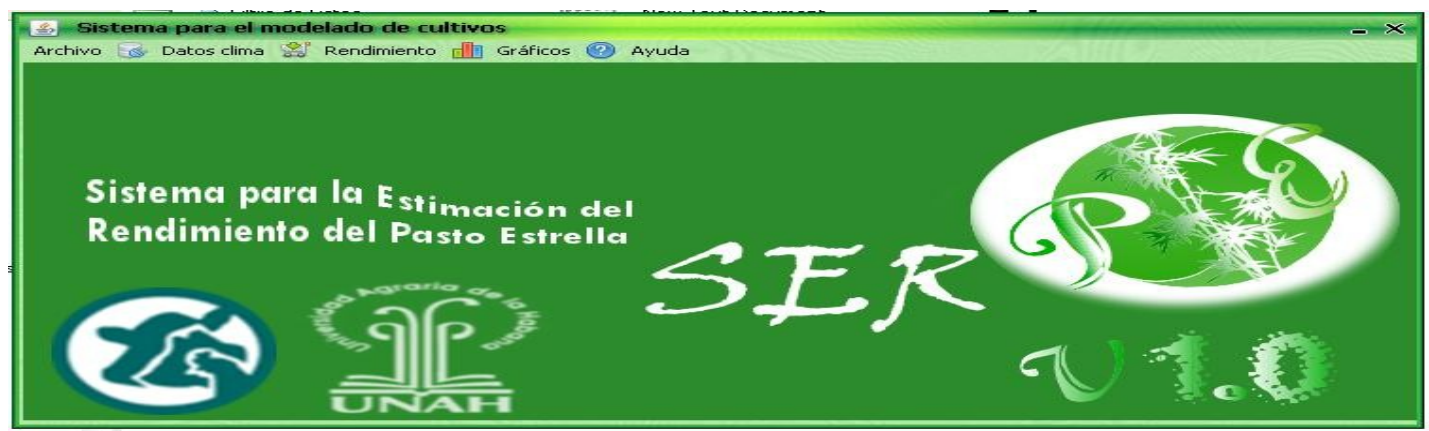

Fuente: Elaboración propia 
En la figura 6 se muestra la captura de datos, para estimar RMSA con efectos climáticos.

Figura 6. Captura información para estimar el RMSA con efecto ambiental.

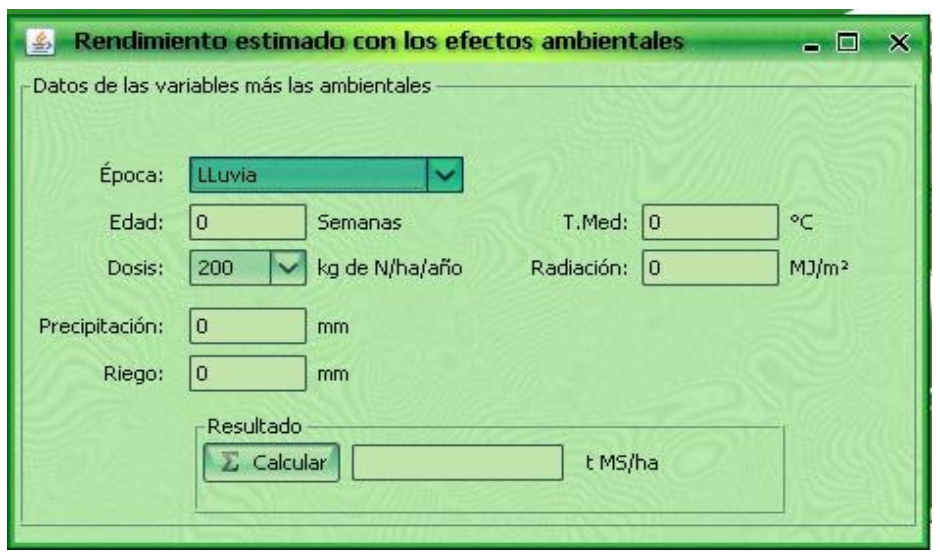

Fuente: Elaboración propia.

Como se puede observar, en la figura 7 se expone la captura de datos, para estimar RMSA sin efecto del clima.

Figura 7. Captura información para estimar el RMSA sin efecto ambiental

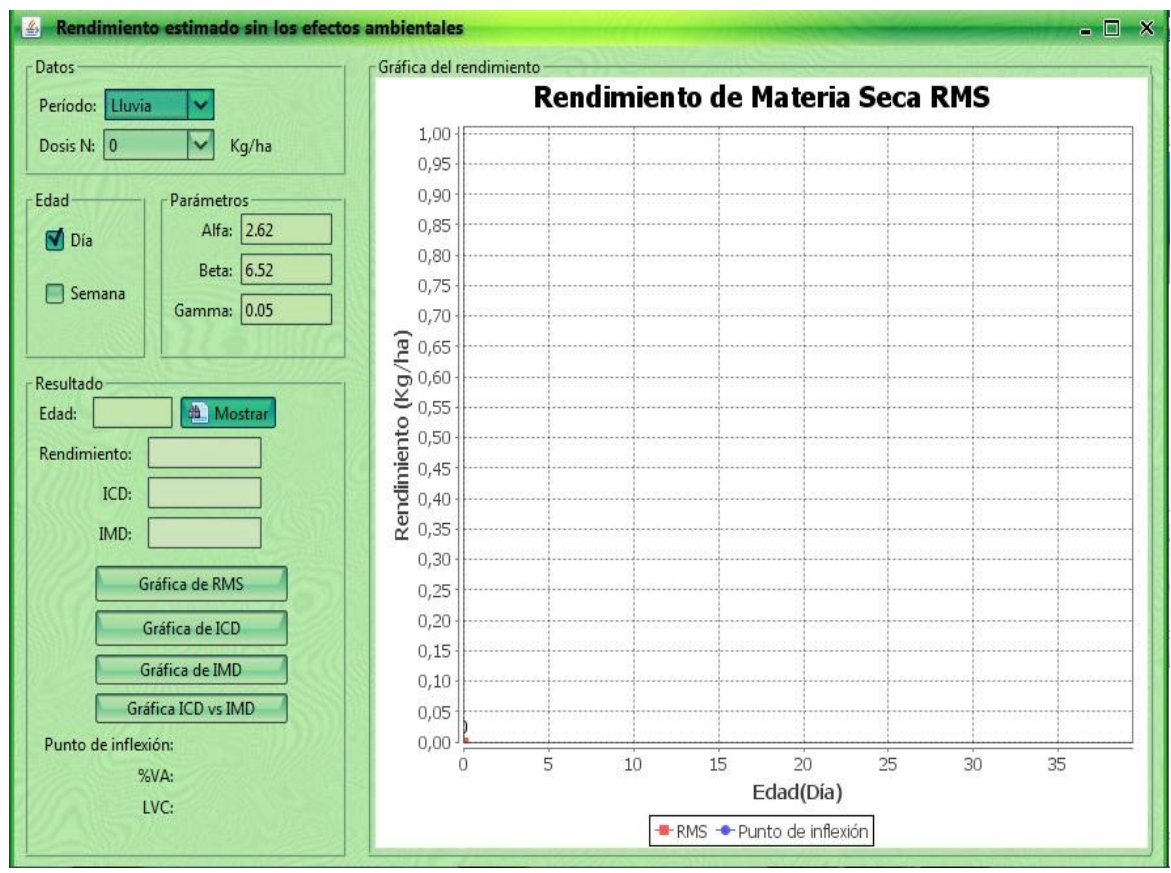

Fuente: Elaboración propia.

La figura 8, muestra las curvas de incrementos corriente medio, diario y de crecimiento (Gompertz), para esta especie. 
Figura 8 Curvas de incrementos medio, diario y de crecimiento para el pasto estrella.
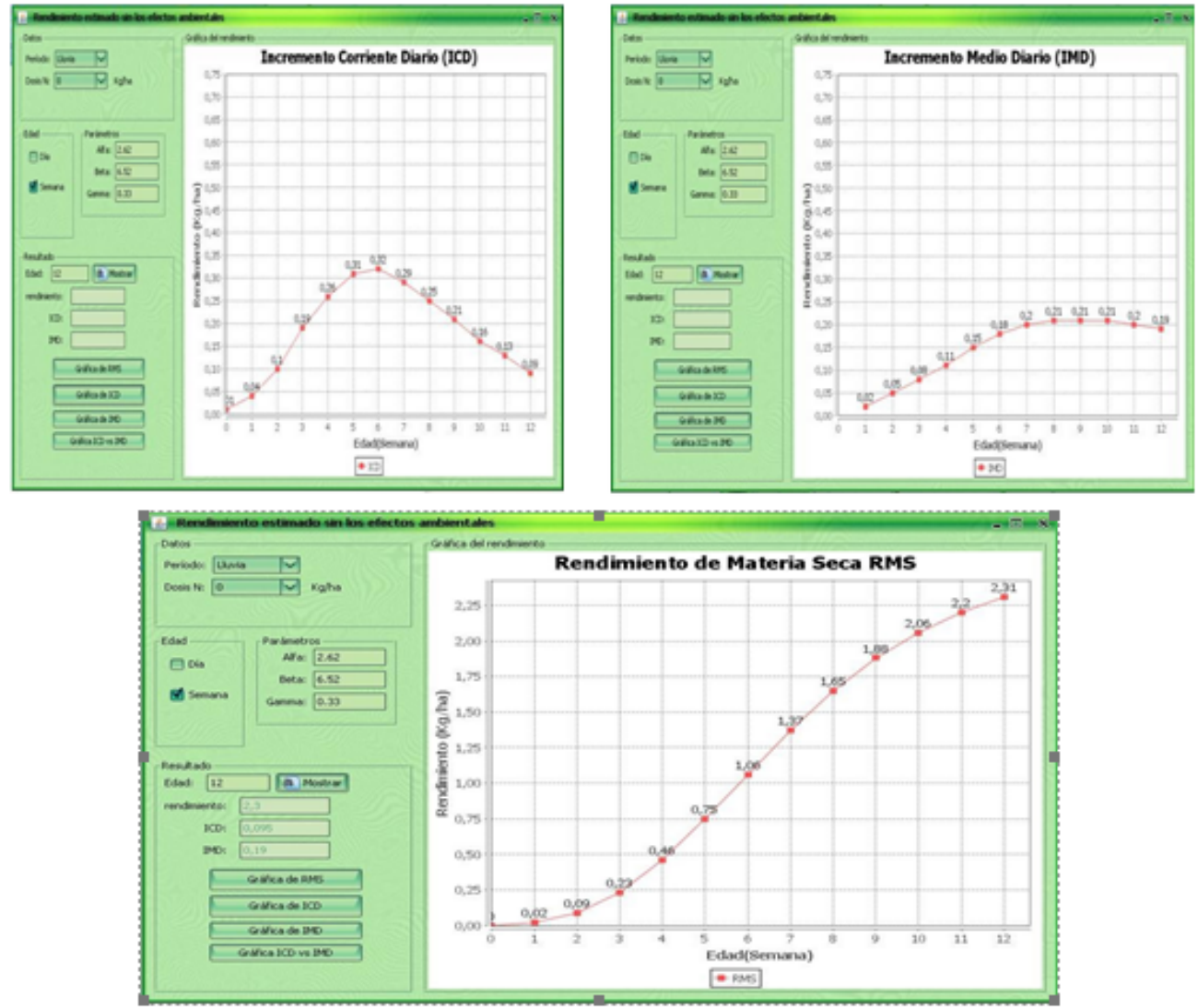

Fuente: Elaboración propia.

En la figura 9 se aprecia la edad óptima en que debe ser cosechado el pasto estrella desde el punto de vista matemático.

Figura 9. Edad óptima de cosecha del pasto estrella desde el punto de vista matemático.

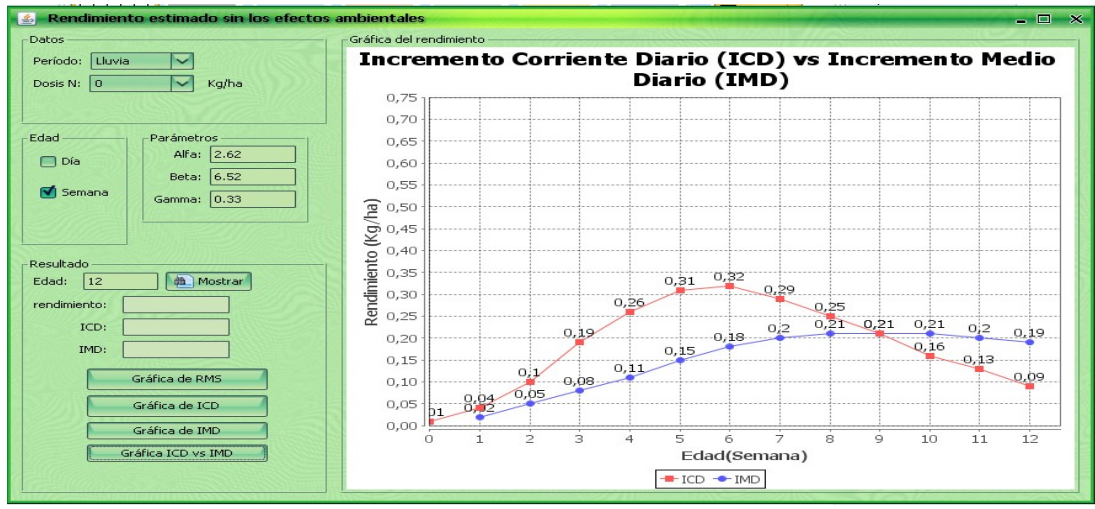

Fuente: Elaboración propia. 
Por otra parte, la utilización por los productores de herramientas de ayuda en la toma de decisiones a través de la modelación y simulación, ha sido tradicionalmente baja (McCown, 2002). En el caso de los modelos de crecimiento de pastos, Wight y Hanson (1993) identificaron tres elementos fundamentales de la no utilización de estos modelos: en primer lugar, la escasa disponibilidad de datos de clima, la cual es resuelta en este modelo con la utilización de la base de datos meteorológica.

En segundo lugar, la escasa disponibilidad de los datos de suelos y en tercer lugar el número de variables de estado que están presentes en otras aplicaciones y deben ser inicializadas.

Otro aspecto que conspira en la utilización de los modelos es la dificultad de interpretar y aplicar los resultados de estos, mencionada como otra posible explicación a la limitada utilización de los modelos como herramientas de ayuda en la toma de decisiones (Wight y Hanson, 1993).

Por otro lado, en función de la objetividad de los resultados, es recomendable configurar el sistema de forma que se ajuste a las especificidades de la unidad que se evalúe, por ejemplo, que la información sobre la frecuencia de corte, las dosis de $\mathrm{N}$ y sobre todo la información climática, sean lo más precisa posible, para obtener razonables estimaciones de la producción de biomasa.

El software obtenido posee importantes características desde el punto de vista de la ingeniería de software, tales como consistencia, eficiencia, integridad, flexibilidad, portabilidad y seguridad entre otras, todas muy deseables en los programas de aplicación (Menner, 1995; Senn, 1998), su proceso de validación no puede ser considerado totalmente conclusivo.

Lo anterior se debe, fundamentalmente a las características propias de la temática que aborda el software, la concepción del diseño que fue empleada en correspondencia con los objetivos propuestos e incluso, a la no existencia en Cuba de ningún otro programa de aplicación a la estimación de la biomasa para el pasto estrella.

\section{Conclusiones.}

- Se obtuvo una base de datos de variables climáticas que contiene dos escenarios meteorológicos diferentes para el planeta, la misma se incluyó en la herramienta computacional elaborada para simular el rendimiento de materia seca acumulada bajo diferentes condiciones ambientales.

- El modelo Gompertz ajustó los datos para el pasto estrella (C. nlemfuensis) con elevados coeficientes de determinación que fluctuaron entre $99 \%$ para los períodos poco lluvioso y lluvioso respectivamente.

- El momento óptimo de utilización del forraje fue variable y se debe realizar en el punto donde se interceptan las curvas de Incrementos corriente diario y medio. 
- Las edades óptimas de cosecha para la dosis $0 \mathrm{~kg}$ de nitrógeno obtenidas desde el punto de vista matemático resultaron 32,3 y 37,5 días para los períodos poco lluvioso y lluvioso respectivamente. Mientras que, para $200 \mathrm{~kg}$ de nitrógeno, se obtuvieron 44,6 y 30,4 días para ambos períodos estacionales.

- Se encontró que un incremento de la temperatura media del planeta en 2 y $4{ }^{\circ} \mathrm{C}$. tendría efectos negativos en el rendimiento de materia seca acumulada de este cultivo fundamentalmente en el período poco lluvioso.

\section{Referencias bibliográficas.}

Adams R. M., Hurd B. H., Lenhart S. y Leary, N (1998). Effects of global climate change on agriculture: an interpretative review. Climate Research 11(1):1 9-30.

Agudelo, D.A., Cerón, M.F. y Restrepo, L. F. (2008). Modelación de funciones de crecimiento aplicadas a la producción animal. Rev. Col. Cienc. Pec. 21: 39582_misconceptions.pdf. [Consulta 25 de enero 2019].

Anon, (1994). Situación actual de las investigaciones que se desarrollan en el Pastoreo Racional Voisin en el Instituto de Ciencia Animal (MIMEO) 30 p.

Banco Mundial (2015). Lima Perú 5 al 12 octubre 2015. Reunión Anual del BM y del FMI. http://www.bancomundial.org/es/topic.

Bárcena, A (2010). Restricciones estructurales del desarrollo en América Latina y el Caribe: una reflexión postcrisis; Revista CEPAL 10 0, abril 2010.pg 7 - 28.

Bárcena, A, Prado, A., Samaniego, JL. y Pérez, R. (2014).La economía del cambio climático en América Latina y el Caribe. Paradojas y desafíos del desarrollo sostenible. Cepal $98 \mathrm{pp}$.

Barker, D.J., Ferraro, F.P., La Guardia, R., Mark, R., Lopes, F., \&Albrecht, K.A. (2010). Analysis of Herbage Mass and Herbage Accumulation Rate Using Gompertz Equations. AgronomyJournal, Volume 102, Issue 3. Disponible en: http://es.slideshare.net/ [Consultado: 4 de septiembre 2017].

Black Jr CC (1973). Photosynthetic carbon Æxation in relation to net CO2 uptake. Annual Review of Plant Physiology 24, 253 \pm 286.

Campbell, Jayaka D., Taylor, M.A., Stephenson, Tannecia S., Watson, R.A. and Whyte Felicia S. (2011). Future climate of the Caribbean from a regional climate model. Int. J. Climatol. 31: 1866-1878 (2011). 
Del Pozo, P. P. y Herrera, R. S. (1995). Modelado del crecimiento del pasto estrella (Cynodonnlemfuensis). 1. modelo multiplicativo con control de la curva de crecimiento y los efectos ambientales. Rev. Pastos y Forrajes 18(2):171177

Di Rienzo, J. A. 2011. Análisis de regresión. Disponible en: http://sites.google.com/site/dirienzojulio [Consultado: 6 de agosto 2018].

Díaz, D. (2007). Evaluación agronómica de nuevas variedades Pennisetumpurpureum en condiciones de sequía el Valle del Cauto. Tesis en opción al Título Académico de Master en Pastos y Forrajes. Universidad de matanzas "Camilo Cienfuegos, 84 p.

FAO 56 (1997). Evapotranspiración del cultivo. Guías para la determinación delos requerimientos de agua de los cultivos. Estudio FAO Riego y Drenaje. ISSN 02545293.

Friedrich, T. (2014). Producción de alimentos de origen animal. Actualidad y perspectivas. Rev. Cuban. Cienc. Agríc. 48:5

García, A. (2011). Sistema para integrar la operación de las Centrales Telefónicas de diferentes proveedores existentes en todo el país. Trabajo de Diploma. Mayabeque, Cuba, Universidad Agraria de La Habana. hh. 23-25.

Hernández, N., Soto. F. y Caballero, A. (2009). Modelos de simulación de cultivos. Características y usos. Cultivos Tropicales 30:1. Disponible en: http://scielo.sld.cu/ [Consultado: 3 de agosto de 2019].

Herrera, R.S (2009). Mejoramiento de Pennisetumpurpureum en Cuba. Rev. Cubana Cienc. Agríc. 43:345.

IPCC (2001).Climate Change 2001: The Scientific Basis. Contribution of Working Group I to the Third Assessment Report of the Intergovernmental Panel on Climate Change (IPCC). Houghton JT, Ding Y, Griggs DJ, Noguer M, van der Linden PJ, Xiaosu D (Eds.). Cambridge University Press. Cambridge, RU. 944 pp.

IPCC (2015). Special Report on Emissions Scenarios. IPCC Working Group III (WGIII). 35 pp.

Jay, O (2012). Metodología para la comparación de tratamientos en modelos de regresión no lineal aplicados a procesos biológicos. Tesis presentada en opción al título de Doctor en Ciencias Veterinarias, Instituto de Ciencia Animal, La Habana, Cuba. 100 p. 
Jones R. G., Noger M., Hassell D. C., Hudson D., Wilson S., Jenkins G., and Mitchell, J., (2004). Generating high resolution climate change scenarios using PRECIS. Met Offrice Hadley Center, Exeter, UK, 40 pp.

Kiviste, A. J., G., Álvarez, A., Rojo, A., Ruíz, D. (2002). Funciones de crecimiento de aplicación en el ámbito forestal. Ministerio de Ciencia y Tecnología. Instituto de Investigaciones y Tecnología Agraria y Alimentaría. Madrid. 190 p.

Leary, N., Kulkarni, J. y Seipt, C. (2007). Assessment of impacts and adaptation to climate change, Final Report of the AIACC Project, UNEP, Washington.

McCown, R.L. (2002). Changing systems for supporting farmers' decisions: problems, paradigms, and prospects. Agricultural Systems 74: 179-220.

Menner, W.A. (1995). Introduction to modeling and simulation. Johns Hopkins APL Technical Digest. 16(1): 6-17.

Montgomery, D. C., Peck, E. A. y Vining, G. G. (2005). Introducción al análisis de regresión lineal. Compañía Editorial Continental. México. 588 pp

Paz, Y. y Bárcenas, Y. (2011). Sistema de gestión semántica de información geográfica. Trabajo de Diploma. Mayabeque, Cuba, Universidad Agraria de La Habana. h. 24.

Páez, R. L. y Rodríguez, E. (2012). Sistema de extración de términos en el dominio de la informática. Trabajo de Diploma. Mayabeque, Cuba, Universidad Agraria de La Habana. h. 29.

PCC (2011). Lineamientos de la política económica y social del Partido y la Revolución. Partido Comunista de Cuba, La Habana, 38 pp.

PCCC (2015). Programa Cambio Climático. X Convención Internacional Sobre Medio Ambiente $\quad \mathrm{y}$ Desarrollo en: http://www.cubambiente.com/Programa\%20de\%20Cambio\%20Clim\%C3\%A1t ico.pdf.

Puerto, Y. y Mejías, A. (2012). Sistema de gestión de trámitesde salidas al extranjero. Trabajo de Diploma. Mayabeque, Cuba, Universidad Agraria de La Habana. h. 8.

Ramos, N., Herrera, R. S. y Curbelo, F. (1979). Reseña descriptiva del kinggrass. Instituto de Ciencia Animal. Cuba. p.5.

Ramos, N., Herrera, R.S., Schalitz, G. y Curbelo. F. (1995). Estudio del rendimiento y calidad de gramíneas templadas evaluadas en Cuba. Rev. cubana Cienc. agríc. 29:109 
Rodríguez, J., Gutiérrez, E., Rodríguez, H (2010). Dinámica de sistemas de pastoreo. Editorial Trillas. México. 272 p.

Rodríguez, L (2015). Modelación y simulación de la producción de biomasa de Pennisetum Purpureum Schumvc. kinggrass y su aplicación en la alimentación animal. Tesis en opción al grado científico de Doctor en Ciencias Veterinarias. Instituto de Ciencia Animal. Mayabeque. Cuba.

Rodríguez, Y. (2015a). Sistema informático para el análisis y gestión de la entrega y tenencia de tierra en el municipio de Madruga. Trabajo de Diploma. Mayabeque, Cuba, Universidad Agraria de La Habana. h. 39.

Senn, J.A. (1998). Analysis \& Design of Information Systems. McGraw-Hill Inc.,USA. 988 p.

Travieso, M. y Rodríguez, H. (2015). Sistema de información nacional para el control de la inspección estatal. Trabajo de Diploma. Mayabeque, Cuba, Universidad Agraria de La Habana. h. 18.

Wight, R.J., Hanson, C (1993). Making models easy to use. Proceedings of the XVII International Grassland Congress, 769-770.

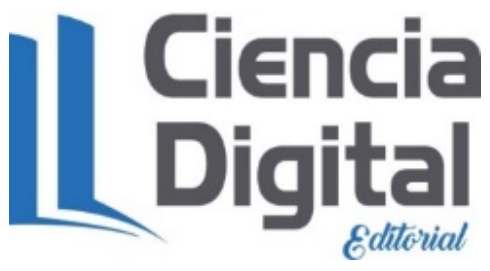




\section{Para citar el artículo indexado}

López Rodríguez, J. L., \& Larduet Vicet, R. (2020). Modelación y simulación del rendimiento del pasto estrella (C.nlemfuensis) bajo diferentes condiciones de manejo y escenarios climáticos. AlfaPublicaciones, 2(3), 21-42. https://doi.org/10.33262/ap.v2i3.33

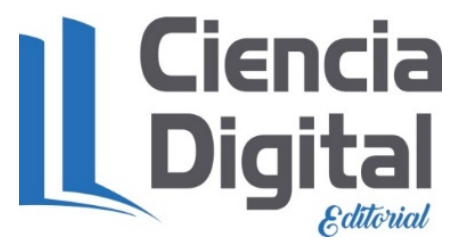

El artículo que se publica es de exclusiva responsabilidad de los autores y no necesariamente reflejan el pensamiento de la Revista Alpha Publicaciones.

El artículo queda en propiedad de la revista y, por tanto, su publicación parcial y/o total en otro medio tiene que ser autorizado por el director de la Revista Alpha Publicaciones.
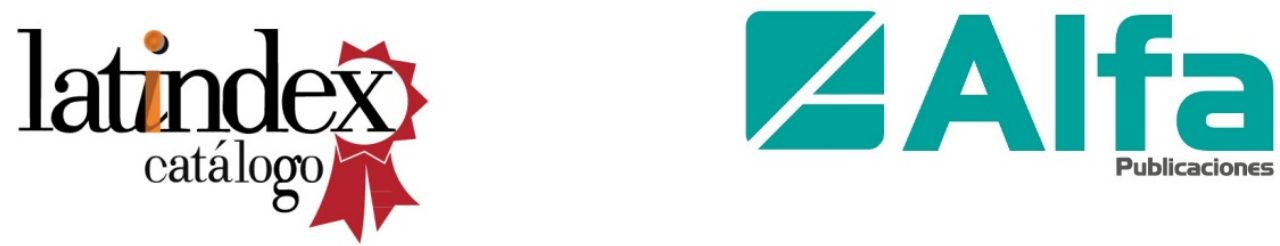